\title{
Bibliometría para la evaluación de la actividad científica en ciencias de la salud
}

\author{
Pablo Juárez-Rolando ${ }^{1, a, b}$.
}

\begin{abstract}
RESUMEN
Este trabajo, describe la importancia de la investigación bibliométrica para la evaluación de la actividad científica en Ciencias de la Salud, considerando en la misma la producción científica Latinoamericana. Un nuevo conocimiento, producto de la investigación científica, adquiere valor cuando se publica y posteriormente es aplicado en el campo específico del estudio, contribuyendo al desarrollo de la sociedad; en este sentido, los resultados de estudios bibliométricos han adquirido una importancia creciente en la elaboración de la políticas de investigación científica y su gestión en el área de la salud.
\end{abstract}

PALABRAS CLAVE: Bibliometría, ciencias de la salud, actividad científica.

\section{Bibliometrics for evaluation of scientific activity in health sciences}

\begin{abstract}
SUMMARY
This paper describes the importance of bibliometric research for the evaluation of scientific activity in health sciences, and considers the Latin American scientific output. New knowledge, products of scientific research has value when published, and subsequently applied in the specific field, contributes to development of society. In this sense, the results of bibliometric studies become increasingly important, in the development and management of scientific policy in the area of health.
\end{abstract}

KEYWORDS: Bibliometrics, health sciences, scientific activity.

\section{INTRODUCCIÓN}

La publicación de un trabajo científico, es el modo más efectivo de transmitir un conocimiento obtenido como consecuencia de la investigación clínica, experimental o de la experiencia personal. Las revistas permiten generar el cuerpo de información, son vehículo de confrontación de opiniones, son su foro de expresión (1).

Evaluar el impacto de la investigación, requiere la medición del conocimiento. Sin embargo, el conocimiento no puede ser medido directamente.
Pueden ser observadas y medidas, las expresiones del conocimiento, como papers, patentes y estudiantes formados (2).

En el sistema científico, la tarea de evaluar los nuevos conocimientos y los resultados de la investigación, tiene una importancia capital. Se evalúan los autores y sus nuevas ideas, la difusión y el impacto de estas ideas, así como el valor de las publicaciones en que se dan a conocer, factores que repercuten en el valor de la política científica de un país y en los rumbos que cabe atribuirle. La evaluación supone un análisis de la medida en que

${ }^{1}$ Unidad de Actividad Académica "Metodología de la Investigación Documental”, Carrera de Posgrado Magister en Investigación en Ciencias de la Salud, Facultad de Odontología. Universidad Nacional del Nordeste. Corrientes, Argentina.

a Dictante ; ${ }^{b}$ Profesor Titular de la Asignatura Fisiología Humana (Módulo Morfofunción II). 
las actividades han alcanzado objetivos específicos (3). La evaluación, además, permite planificar y gestionar la investigación, a través de instituciones cuyos grupos la llevan a cabo y se benefician de las ayudas económicas provenientes de los presupuestos de la administración. Los resultados (output) de la investigación, se dan a conocer al resto de la comunidad de investigadores por medio de las publicaciones científicas, sobre todo a través de los artículos publicados en revistas científicas, con el propósito de que esta comunidad contraste, verifique o rechace el valor de esa investigación $(4,5)$. La bibliometría, como disciplina tiene desde su inicio dos grandes ámbitos de desarrollo y aplicación; por una parte, la respuesta al estudio de la ciencia y la evolución de la producción científica y, por otra, la gestión editorial. Esta disciplina estudia empíricamente la actividad científica generada por los autores y grupos de colaboración, a través del producto final de la investigación, el artículo científico (6).

El objetivo de este trabajo fue definir la importancia y estrategias de la bibliometría para la evaluación de la actividad científica en las ciencias de la salud y analizar la situación de la bibliometría latinoamericana.

\section{Importancia de la bibliometría}

En las denominadas Ciencias de la Vida y de la Salud, la cuantificación y posterior valoración de la producción científica escrita ocupa un lugar preeminente. En la salud confluyen lo biológico y lo social, el individuo y la comunidad, la política social y la económica. Como toda tradición viva, la salud debe estar en continuo proceso de construcción y renovación (7).

El estudio de la producción científica en un área temática determinada sigue siendo un buen indicador del avance de las investigaciones y la generación de nuevos conocimientos. El análisis bibliométrico no solo permite examinar retrospectivamente cómo se han logrado y dado a conocer los avances científicos y evaluar el potencial de investigación de las instituciones involucradas, sino que además permite caracterizar el desarrollo de disciplinas científicas y sus líneas de investigación, y las publicaciones científicas en un área del conocimiento, su obsolescencia y dispersión (8).

La bibliometría, nos permitirá indagar los cambios en la actividad de investigación, compromiso relativo y colaboraciones entre regiones con similares cuidados de salud de la población y parámetros de desarrollo $(9,10)$.
Esta información, es una herramienta útil para el análisis de la inversión en ciencia y tecnología de la salud, que complementada con otros indicadores socioeconómicos y la opinión de expertos, permitirán la toma de decisiones para la elaboración de las correspondientes políticas públicas sobre investigación y desarrollo, que impulsen el proceso innovador y fomenten la investigación en el sector sanitario (11).

Se ha demostrado que la aplicación de métodos bibliométricos, ha sido satisfactoria para el desarrollo de iniciativas de salud públicas para enfermedades específicas, como la diabetes y tuberculosis $(12,13)$.

La bibliometría ha adquirido una importancia creciente en la política y la gestión de la ciencia en la última década y es específicamente en el ámbito de la evaluación de la investigación en el que desempeña un papel destacado (2). La evaluación debe generar insumos para proponer otras formas en que el conocimiento científico y sus aplicaciones apoyen las políticas y programas de los gobiernos, así como posibilitar la detección de carencias con respecto a indicadores en las áreas de la ciencia y la tecnología que sea necesario diseñar e incluir (4).

Para que los resultados de las evaluaciones posean una utilidad social real y su implementación sea más factible, deben de incorporar, desde su diseño, a los actores sociales para los cuales es relevante este proceso, sean estos investigadores, empresarios, funcionarios públicos, organizaciones ciudadanas o representantes de comunidades rurales organizadas (14).

Cuando se considera aisladamente, se podría argumentar que la bibliometría es altamente reduccionista, una puntuación cuantitativa, numérica, que no es representativa del rendimiento o la capacidad de un investigador. Sin embargo, la bibliométria tiene una gran utilidad, porque en términos pragmáticos representa una fuente de datos precisos y de obtención relativamente simple (15).

\section{Estrategias de la bibliometría}

Existen tres tipos de estudios bibliométricos: Descriptivos, relacionales y evaluativos. Los descriptivos hacen hincapié en los rasgos característicos del documento. Incluye el estudio de la cantidad de publicaciones en un campo dado o la productividad de literatura en el campo con el fin de comparar la investigación en diferentes instituciones/ países así como en diferentes períodos. La bibliometría relacional pretende clarificar las relaciones dentro de la 
investigación, como la estructura cognitiva de los campos de investigación, la aparición de nuevos frentes de investigación, o los patrones nacionales o internacionales de coautoría. La bibliometría de evaluación busca valorar el impacto de los trabajos académicos, y compara las contribuciones relativas de dos o más individuos o grupos $(16,17)$.

\section{¿Cómo se evalúa la producción científica del área salud?}

Se aplican básicamente, indicadores bibliométricos combinados de producción, uso y visibilidad bajo los criterios de impacto, calidad, liderazgo, al igual que en las otras ciencias.

Teniendo en cuenta, los diferentes indicadores bibliométricos al realizar una revisión, como por ejemplo, de alguna revista, se traza la fecha que se va a estudiar o el intervalo de tiempo y posteriormente se buscan los trabajos originales (o todos los trabajos o publicaciones de la revista), y de aquí se toman los datos pertinentes. Ej.: autor, número de firmantes, país de procedencia, índice de productividad, etc., (18).

Las revisiones, se clasifican en macro, meso o microestudios.; en macro se engloba el estudio de la producción científica de un país, ciudad o provincia; en meso, a instituciones o grupos investigativos, y micro, a investigadores o revistas específicas (19).

A nivel micro, los tres indicadores bibliométricos más conocidos son: el factor de impacto, el índice de inmediatez y la vida media de los artículos científicos (20). El más utilizado, es el factor de impacto, siendo útil al escoger una revista para publicar un artículo o para suscribirse. Aunque, en ocasiones se equipara alto factor de impacto con mayor calidad, mide específicamente la visibilidad y la difusión de los trabajos publicados en estas revistas más que la calidad científica de los mismo(21).

Así, la selección de bases de datos adecuadas, nos permiten acceso a publicaciones científicas de calidad importante y la bibliometría, nos permite calificar y determinar el impacto de dichas publicaciones en la comunidad científica y el valor que representan (22).

El Institute for Scientific Information (ISI), calcula y publica los factores de impacto, el ISI es una institución privada, creada en 1955, como una herramienta para recuperar bibliografía, en su revista de reporte de citas: Journal Citation Report, y mediante la gestión de la importante base de datos Science Citation Index, con revistas de casi todas las áreas de conocimiento, a nivel mundial; pero, muestra una falta de cobertura amplia, hacia la ciencia de los países en vías de desarrollo (23), su nombre actual es Thomson Reuters Scientific y su producto estrella actual es el Web of Knowledge.

Actualmente, realizar un análisis bibliométrico en Latinoamérica y el Caribe es más fácil pues existen bases de datos hispanoamericanas (SciELO, LILACS, Redalyc y Publindex), donde encontramos información de indicadores bibliométricos que nos permiten tomar datos biliométricos y procesarlos (24).

Las revistas de "acceso abierto" son una nueva opción en la difusión, registro y análisis de la evidencia científica. El hecho de que estos documentos están ampliamente y libremente disponibles, asegura citas óptimas y posibilita una mayor profundización en los estudios bibliométricos $(15,25)$.

\section{Bibliometría Latinoamericana}

Independientemente de los informes generales, que se publican regularmente sobre indicadores en Ciencia y Tecnología del mundo, no hay muchos estudios bibliométricos que analicen las tendencias latinoamericanas, en el ámbito específico de las Ciencias de la Salud.

Uno de los primeros antecedentes, se obtuvo del Boletín de la Oficina Sanitaria Panamericana, con un análisis bibliométrico en la revista Archivos Latinoamericanos la cual en el año 1995 publicó: "La influencia y el impacto de las publicaciones de la OPS en la producción científica sobre salud en América Latina y el Caribe" (26).

Desde esa fecha, se ha venido observando un aumento de los estudios bibliométricos en Ciencias de la Salud, como evidencian trabajos en su gran mayoría sobre estudios específicos sobre una especialidad o disciplina (27-32).

Estos estudios, permitirán generar una política de investigación latinoamericana, que redunde en el beneficio de las diversas profesiones de las Ciencias de la Salud.

Hasta el momento, son moderados los estudios bibliométricos que permiten examinar con un criterio cuantitativo, retrospectivo e integral la producción científica sobre salud pública en América Latina y el Caribe. Uno de los pioneros fue Macías-Chapula, quién 
presentó los resultados de un análisis bibliográfico de la producción científica sobre salud pública, cuya finalidad fue construir un modelo de comunicación científica en este campo, que sirva de apoyo para que investigadores, gestores y trabajadores de la salud pública puedan tomar las decisiones y las acciones necesarias (33).

Sin embargo, durante el período 1996-2010, Latinoamérica tiene una producción superior a nivel mundial, en categorías temáticas vinculadas con la salud pública. En la categoría epidemiología, la región latinoamericana produce artículos en proporciones muy similares la producida en el resto del mundo. En la categoría políticas de salud, la región comienza a tener valores de especialización por encima del mundo a partir del 2006, con una tendencia al crecimiento sostenido y una clara prevalencia de la producción cubana (34).

Estudios realizados sobre la transferencia de información y conocimiento, en el contexto de un sistema de salud, demostraron que el conocimiento científico generado en salud pública, es publicado, organizado y administrado para ser utilizado por otros investigadores, pares o profesionales de la salud del sistema y para su transferencia a la población (35).

En el mismo campo, se vienen realizando análisis bibliométricos sobre "inequidades en salud", dentro y entre países de Latinoamérica y el Caribe. Las publicaciones comprendidas en el período 1971-2000, mostraron un rápido crecimiento en los últimos años. Brasil, Chile, México contribuyeron sobre todo con investigación empírica, mientras que Ecuador y Argentina produjeron más estudios conceptuales (36).

La búsqueda de la equidad, calidad y eficiencia en los sistemas y servicios de salud requiere de conocimientos de calidad aportados por las investigaciones que contribuyan a ese objetivo. Esto es válido para todos los países y aun con mayor prioridad para países con escasos recursos que necesitan avanzar en forma acelerada hacia la equidad y las metas del milenio $(37,38)$.

\section{CONCLUSIONES}

La bibliometría, es una herramienta útil para objetivar los productos de la investigación científica en Ciencias de la Salud, volviendo tangible procesos, que nos permiten la toma de decisiones y la implementación de políticas de salud.
Es preciso contextualizar los resultados de los análisis bibliométricos, atendiendo a los aspectos biomédicos, ecológico-ambientales y socio-culturales, e integrando toda la información para su gestión.

Conflicto de intereses: El autor indica que no tiene conflicto de interés que declarar.

\section{REFERENCIAS BIBLIOGRAFICAS}

1. De Vito EL. Algunas consideraciones en torno al uso del Factor de Impacto y de la Bibliometría como herramienta de evaluación científica. RAMR. 2006; 6(1):37-45.

2. Castiel LD, Sanz-Valero J. Política científica: manejar la precariedad de los excesos y desnaturalizar la ideología "publicacionista" todopoderosa. Salud Colect. 2009; 5(1):511. DOI: $10.1590 / \mathrm{S} 1851-82652009000100001$.

3. Milanés Y, Pérez Y, Peralta MJ, Ruiz ME. Los estudios de evaluación de la ciencia: aproximación teórico-métrica. ACIMED. 2008; 18(6): 0-0.

4. Spinak E. Indicadores cienciométricos. ACIMED. 2001; 9 (S4): 16-18.

5. Salager-Meyer F. Scientific publishing in developing countries: Challenges for the future. JEAP. 2008; 7(2):121132. DOI: doi:10.1016/j.jeap.2008.03.009.

6. Casterá VT, Sanz-Valero J, Juan-Quilis V, Wanden-Berghe C, Culebras JM, García de Lorenzo y Mateos A. Estudio bibliométrico de la revista Nutrición Hospitalaria en el periodo 2001 a 2005: Parte I, análisis de la producción científica. Nutr Hosp. 2008; 23(5):469-476.

7. Rousseau R. Journal Evaluation: Technical and Practical Issues. Libr Trends 2002; 50(3):418-439.

8. Sanz-Valero J, Tomás Casterá V, Wanden-Berghe C. Estudio bibliométrico de la producción científica publicada por la Revista Panamericana de Salud Pública 1997 a 2012. Rev Panam Salud Publica. 2014;35(2):81-8.

9. Traynor M, Rafferty AM. Bibliometrics and a culture of measurement. J Adv Nurs. 2001; 36:167-8.

10. Smith K, Marinova D. Use of bibliometric modelling for policy making. Math Comput Simul. 2005; 69:177-87. DOI:10.1016/j.matcom.2005.02.027.

11. Alvis-Guzmán N, De La Hoz-Restrepo F. Producción Científica en Ciencias de la Salud en Colombia, 1993-2003. Rev de Salud Pública (Bogotá, Colombia). 2006;8(1):25-37.

12. Arunachalan S, Gunasekaran S. Tuberculosis research in India and China: from bibliometrics to research policy. Curr Sci. 2002; 82(8):933-47.

13. Arunachalan S, Gunasekaran S. Diabetes research in India and China today: from literature-based mapping to healthcare policy. Curr Sci. 2002;82(9):1086-97.

14. González MV, Molina M. La evaluación de la ciencia y la tecnología: revisión de sus indicadores. ACIMED. 2008;18(6):1-19. (Fecha de consulta: 2 Junio 2016);Disponible en: http://bvs.sld.cu/revistas/aci/ vol18_6_08/aci031208.htm 
15. Davidson PM, Newton PJ, Ferguson C, et al. Rating and ranking the role of bibliometrics and webometrics in nursing and midwifery. Scientific World J. 2014. doi:10.1155/2014/135812

16. Borgman CL, Furner J. Scholarly communication and bibliometrics. Annual Review of Information Science and Technology 2002; 36(1):3-72. doi: 10.1002/aris.1440360102.

17. Thelwall M. Bibliometrics to webometrics. Journal of Information Science 2008; 34 (4):605-621. doi: 10.1177/0165551507087238.

18. Chalmers I. Evaluation "payback” on biomedical research. Biomedical funding decisions should be audited. BMJ [en línea]. 2000;321(7260):566.

19. Franks A, Simoes E, Singh R, Sajor Gray B. Assessing prevention research impact a bibliometric analysis. Am J Prev Med. 2006; 30(3):211-216.

20. Caballero-Uribe CV, Cuello M, Lubo A, et al. El factor de impacto en la evaluación de las revistas biomédicas. Salud Uninorte. 2006;22(2):92-104.

21. Johnstone MJ. Journal impact factors: implications for the nursing profession: original article. Int Nurs Rev. 2007;54(1):35-40.

22. Castrillón-Estrada JA, García JC, Anaya M, Rodríguez D, De la Rosa D, Caballero-Uribe CV. Bases de datos, motores de búsqueda e índices temáticos: herramientas fundamentales para el ejercicio médico. Salud Uninorte. 2008;24(2):96-119.

23. Macías-Chapula C, Mendoza-Guerrero JA, Rodea-Castro IP, Gutiérrez-Carrasco A. Construcción de una metodología para identificar investigadores mexicanos en bases de datos del ISI. Rev Espa de Doc Cienti. 2006; 29(2):220-238.

24. Dávila M, Guzmán R, Macareno H, Piñeres D, de la Rosa D, Caballero-Uribe CV. Bibliometría: conceptos y utilidades para el estudio médico y la formación profesional. Salud Uninorte. 2009; 25(2):319-330.

25. Torres-Salinas D, Robinson-García N, Aguillo IF. Bibliometric and benchmark analysis of gold open access in Spain: big output and little impact. El profesional de la información. 2016;25(1):17-24. doi: 10.3145/epi.2016. ene. 03

26. Cruz A. Influencia de las publicaciones de la OPS en la producción científica en salud en América Latina y el Caribe. Bol Of Sanit Panam. 1995; 119(6):515-28.

27. Macías-Chapula CA, Sotolongo-Aguilar GR, Madge B, Solorio-Lagunas J. Subject content analysis of AIDS literature, as produced in Latin America and the Caribbean. Scientometrics. 1999;46(3):563-74.

28. Weisinger JR, Vellori-Font E. Latin American Nephrology: scientific production and impact of publications. Kidney Int. 1999;56(4):1584-90.

29. Rodrigues PS, Fonseca L, Chaimovich H. Mapping cancer, cardiovascular and malaria research in Brazil. Braz J Med Biol Res. 2000; 33(8):853-67.

30. Díaz D. Análisis bibliométrico de la revista Archivos Latinoamericanos de Nutrición. An Venez Nutr. 2007; 20(1):22-29.

31. Vernaza-Pinzón P, Álvarez-Bravo G. Producción científica latinoamericana de fisioterapia / kinesiología. Aquichan.
2011;11(1):94-107.

32. Estrada M, Cristancho S. La información científica en la investigación sobre salud mental en la Universidad de Antioquia, Colombia. Rev Cuba Inf Cienc Salud. 2014;25(1):4-23.

33. Macías-Chapula CA. Hacia un modelo de comunicación en salud pública en América Latina y el Caribe. Rev Panam Salud Publica. 2005;18(6):427-38.

34. Arencibia-Jorge R, Vega-Almeida R, Chinchilla-Rodríguez Z, Corera-Álvarez E, Moya-Anegón F. Patrones de especialización de la investigación cubana en salud. Revista Cubana de Salud Pública. 2012;38 (S5):734-747.

35. Macías-Chapula CA. Diseño de un modelo conceptual sobre la transferencia de resultados de investigación en salud pública en Honduras. Salud pública Méx. 2012;54(6):624631 .

36. Almeida-Filho N, Kawachi I, Filho AP, Dachs JNW. Research on Health Inequalities in Latin America and the Caribbean: Bibliometric Analysis (1971-2000) and Descriptive Content Analysis (1971-1995). Am J Public Health. 2003;93(12):2037-2043.

37. Paganini J, Raiher S. Análisis bibliométrico de las publicaciones sobre investigación en servicios de salud. Tendencias y características. Buenos Aires: Universidad Nacional de La Plata,Centro Interdisciplinario para la Salud; 2006 (Citado el 15 de Mayo del 2016). Disponible en: http:// www.inus.org.ar/documentacion/Documentos\%20Tecnicos/ Analisis_bibliometrico_publicaciones_investigacion.pdf

38. Raiher S. Estudio comparativo entre la producción científica latinoamericana y mundial en inequidades en salud durante los años 1999-2008. Tesis presentada para la obtención del grado de Licenciada en Bibliotecología y Documentación

39. La Plata, Argentina: Universidad Nacional de La Plata, Facultad de Humanidades y Ciencias de la Educación; 2010 (Citado el 15 de Mayo del 2016). Disponible en: http:// www.fuentesmemoria.fahce.unlp. edu.ar/tesis/te.361/te.361. pdf

\section{Correspondencia:}

Rolando Pablo Juárez.

Correo electrónico: ropablojuarez@gmail.com

Fecha de Recepción: 15 de abril del 2016. Fecha de aceptación: 06 de junio del 2016. 